\title{
基于柱[5]芳烃的新型双-[1]轮烷的设计与合成
}

\author{
张润沝 $a, b$ 王陈威 $b$ 孙 晶 $a$ 颜朝国 $*, a$ 姚勇 $*, b$ \\ $\left({ }^{a}\right.$ 扬州大学化学化工学院 江苏扬州 225000) \\ ( ${ }^{b}$ 南通大学化学化工学院 江苏南通 226019)
}

\begin{abstract}
摘要 以不同长度的亚烷基二胺单元作为柱 [5]芳烃边缘侧链的一系列柱[5]单酰胺衍生物, 在氯仿中能够形成准 [1]轮 烷. 基于这一中间体准 [1]轮烷的合成, 通过柱[5]单酰胺衍生物和双水杨醛的缩合反应, 成功构建了 8 个柱芳烃的机械 自锁分子(MSMs), 即双-[1]轮烷. 通过多种方法详细地对新合成的双-[1]轮烷进行了研究, 包括 ${ }^{1} \mathrm{H} N \mathrm{NM},{ }^{13} \mathrm{C} N \mathrm{NM}, 2 \mathrm{D}$ NOESY NMR 和 MS 分析, 发现这类新型 MSMs 与 $\mathrm{Cu}^{2+}$ 有较强的识别作用, 两者之间以 $1: 2$ 进行络合.
\end{abstract} 关键词 柱[5]芳烃; 双-[1]轮烷; 准[1]轮烷; 机械互锁分子

\section{Design and Construction of Pillar[5]arene-Based Bis-[1]rotaxane}

\author{
Zhang, Runmiao ${ }^{a, b} \quad$ Wang, Chenwei $^{b} \quad$ Sun, Jing $^{a} \quad$ Yan, Chaoguo $^{*, a} \quad$ Yao, Yong ${ }^{*, b}$ \\ ( ${ }^{a}$ College of Chemistry and Chemistry Engineering, Yangzhou University, Yangzhou, Jiangsu 225000) \\ ( ${ }^{b}$ College of Chemistry and Chemistry Engineering, Nantong University, Nantong, Jiangsu 226019)
}

\begin{abstract}
A series of pillar[5] arene monoamide derivatives with different lengths of alkylenediamine unites as the side chains on the pillar[5]arene's rim can form pseudo[1]rotaxanes. Thus, based on the construction of this intermediate of pseudo[1]rotaxane, eight pillar[5]arene-baed mechanically interlocked molecules (MIMs) of bis-[1]rotaxane were successful constructed through condensation reaction of pillar[5] arene monoamide derivative and salicylaldehyde. The newly synthesized bis-[1]rotaxane was investigated in detail by various methods, including ${ }^{1} \mathrm{H} N \mathrm{NM},{ }^{13} \mathrm{C}$ NMR, $2 \mathrm{D}$ NOESY NMR and MS analysis. It was found that this kind of MIMs has a strong recognition effect with $\mathrm{Cu}^{2+}$, which complexed by $1: 2$.
\end{abstract}

Keywords pillar[5]arene ; bis-[1]rotaxane; pseudo[1]rotaxane; mechanically interlocked molecules

随着冠梄 ${ }^{[1]}$ 作为第一代超分子大环主体化合物的出 现，超分子化学 ${ }^{[2]}$ 逐渐成为许多科学家研究的重要领域 之一, 在纳米科学、材料科学和生物科学等领域得到广 泛应用 ${ }^{[3]}$. 机械互锁分子(MIMs) ${ }^{[4]}$ 已经在超分子化学中 得到了广泛的研究, 为先进的超分子系统 ${ }^{[5]}$ (如轮烷 ${ }^{[6]}$ 、 索烃 ${ }^{[7]}$ 、分子梭 ${ }^{[8]}$ 、开关 ${ }^{[9]}$ 和探针 $\left.{ }^{[10]}\right)$ 的构建提供了无限 的可能, 也使超分子化学适用于分子机器 ${ }^{[1]]}$ 、化学探 针 $^{[12]}$ 和药物输送 ${ }^{[13]}$ 的构建. 虽然 MIMs 的研究近些年快 速发展，但一类新型的机械 MIMs, 即自锁分子(MSMs) 的研究报道却很少, 这是由于 MSMs 的轴和外圈的轮在 一个分子中, 其合成分离较为复杂, 限制了机械自锁分 子的快速发展. 在有限的机械自锁分子中, 准 [1]轮烷是
基本和典型的代表 ${ }^{[14]}$. 在构建机械自锁分子的过程中, 主体提供的空腔大小和性质是最关键的. 作为冠醚、环 糊精、杯芳烃和葫芦腿之后相对较新的大环主体化合物 柱 $[n]$ 芳烃 ${ }^{[15,16]}$, 由于具有富电子空腔、对称的柱状结构 和易于修饰的边缘，已被广泛应用于构建新的超分子聚 合物、分子器件、人工跨膜通道以及化学和物理响应材 料 ${ }^{[17]}$. 柱[5]芳烃是柱芳烃家族中应用最广泛的一类, 因 此, 单官能化柱 [5]芳烃是构建准 [1]轮烷的最通用的候 选化合物 ${ }^{[18]}$. 例如, Ogoshi 等 ${ }^{[19]}$ 报道了基于含有辛基三 甲基铵基团的柱 [5]芳烃的准轮烷，其在氯仿中表现出 自络合的性质. 曹德榕等 ${ }^{[20]}$ 报道了基于单酯化柱 [5]芳 烃的准 [1]轮烷，它可以选择性地结合不同的二卤代烷

\footnotetext{
* Corresponding authors. E-mail: cgyan@yzu.edu.cn; yaoyong1986@ntu.edu.cn Received June 6, 2019; revised July 30, 2019; published online August 7, 2019. Project supported by the National Natural Science Foundation of China (Nos. 21801139, 21871227), the Natural Science Foundation of Jiangsu Province (No. BK20180942), the Natural Science Foundation of Nantong University for High-Level Talent (No. 03083004), and the Large Instruments Open Foudation of Nantong University (No. KFJN1814).

国家自然科学基金(Nos. 21801139, 21871227)、江苏省自然科学基金(No. BK20180942)、南通大学高层次人才启动基金(No. 03083004)、南通大学大 型仪器平台开放基金(No. KFJN1814)资助项目.
} 
烃. 薛敏等 ${ }^{[21}$ 通过单羧酸官能化的柱 [5]芳烃与长链烷 基胺的反应，高产率地制备了基于柱[5]芳烃的 [1]轮烷. 王乐勇等 ${ }^{[22}$ 报道了通过缩合反应形成一个基于芳烃的 互锁准 [1]索烃和准 [1]轮烷(一个包括二氨基烷烃和一个 酸官能化的柱[5]芳烃, 另一个包括二异氰酸酯和胺官 能化的柱 [5]芳烃), 它显示出溶剂和客体的不同响应. 杨英威等 ${ }^{[23]}$ 报道了一种带有咪唑鎓盐基团的单官能化 柱[5]芳烃, 即使在氯仿中高浓度也能形成稳定的准 [1] 轮烷. 近期, 刘育等 ${ }^{[24]}$ 报道了基于几个柱 [5]芳烃的准 [1]索烃, 其中柱芳烃环由两个酰胺基团连接.

我们课题组 ${ }^{[25]}$ 成功合成单官能化柱[5]芳烃席夫碱、 尿素、吡啶亚胺衍生物, 在结构确定过程中, 发现这些 单官能化柱 [5]芳烃在溶液和固体状态下均可以发生自 络合形成较稳定准[1]轮烷. 基于此, 2016 年, 我们 ${ }^{[26]}$ 报 道了一种基于二烯基柱 [5]芳烃的准 [1]轮烷, 并研究了 自锁准 [1]轮烷的稳定性. 最近, 我们课题组 ${ }^{[27]}$ 成功实现 以三联吡啶封端的自锁 [1]轮烷, 并探究了形成 [1]轮烷 的条件. 在本项研究中, 先合成了柱[5]单酰胺衍生物准 [1]轮烷, 然后末端的胺基与双水杨醛类物质 ${ }^{[28]}$ 进行缩 合反应得到目标产物双-[1]轮烷.

\section{1 结果与讨论}

\section{1 目标化合物的合成}

目标化合物基于柱 [5]芳烃的双-[1]轮烷的合成路线 如 Scheme 1 所示. 首先, 进行化合物 1 与丙、丁二胺和 己二胺以 $1: 1$ 化学计量比反应, 得到较为稳定的准 [1] 轮烷. 在制备准轮烷的过程中, 二胺化合物的投料是 $\mathbf{1}$
的 20 倍, 以保证二胺化合物的一个胺基和化合物 $\mathbf{1}$ 反 应. 通过 ${ }^{1} \mathrm{H}$ NMR 研究可以观察到在负方向的化学位移 信号, 表明胺基烷烃链穿入柱 [5]芳烃的空腔内, 然后这 些中间体准轮烷与化合物 $\mathbf{2 a} \sim \mathbf{2 c}$ 经过缩合反应, 以可 观产率获得基于桥联柱 [5]芳烃的双-[1]轮烷 $\mathbf{3 a} \sim \mathbf{3 h}$.

\section{2 目标化合物的表征}

双 [1]轮烷 3a 3h 通过 ${ }^{1} \mathrm{H} N M R 、{ }^{13} \mathrm{C} N M R 、 2 D ~ N M R$ $\left({ }^{1} \mathrm{H}-{ }^{1} \mathrm{H}\right.$ NOESY NMR)、IR 和高分辨质谱 MS 等手段进 行表征. 在席夫碱桥联双柱[5]芳烃 $\mathbf{3 a} \sim \mathbf{3 h}$ 的 ${ }^{1} \mathrm{H}$ NMR 谱图的负场方向, 可以明显观察到一些信号峰. 8 种双 [1]轮烷在负场方向氢的个数如表 1 所示. 图 1 表示 $3 \mathbf{a}$ 在 $\mathrm{CDCl}_{3}$ 中的 ${ }^{1} \mathrm{H} \mathrm{NMR}$ 谱，根据负场中的质子数，将它 们归属于 $3 \mathbf{a}$ 的胺基烷烃链上质子 $\mathrm{H}_{1-4}$, 由于相应的 $\mathrm{CH}_{2}$ 受到柱芳烃空腔的电子云的屏蔽作用从而使化学位移 向高场移动 $(\Delta \delta=-0.07 \sim-2.27)$, 这说明两个胺基烷 烃链穿入柱芳烃的两个空腔中, 表明形成了 [1]轮烷. 这 一结论是与我们课题组之前合成的基于单酰胺功能化 柱 [5]芳烃的 [1]轮烷所得结论一致 ${ }^{[29]}$. 在 2D NOESY $\mathrm{NMR}$ 图谱(图 2)中, 明显观察到已基质子 $\mathrm{H}_{1-4}$ 与 $\mathbf{3 a}$ 的柱 [5]芳烃侧链亚甲基质子 $\mathrm{H}_{\mathrm{a}}$ (图 2, A、B、C、D)、 $\mathrm{H}_{1-4}$ 与 $\mathrm{NH}$ 质子 $\mathrm{H}_{\mathrm{b}}$ (图 2, $\mathrm{E} 、 \mathrm{~F}$ ) 以及 $\mathrm{H}_{1-4}$ 与苯环质子 $\mathrm{H}_{\mathrm{c}}(\mathrm{G} 、 \mathrm{H}$ 、 I、J)之间存在明确的相关信号，这也证实了烷基链穿入 柱芳烃空腔中, 形成了双-[1]轮烷的机械互锁结构. 其 他 7 个基于柱芳烃的双-[1]轮烷同样通过以上手段进行 表征. 通过对比发现双-[1]轮烷中氢在 $\delta 0$ 以下出峰的位 置和个数与烷基二胺的链长 $n$ 有关, 进一步说明柱芳烃

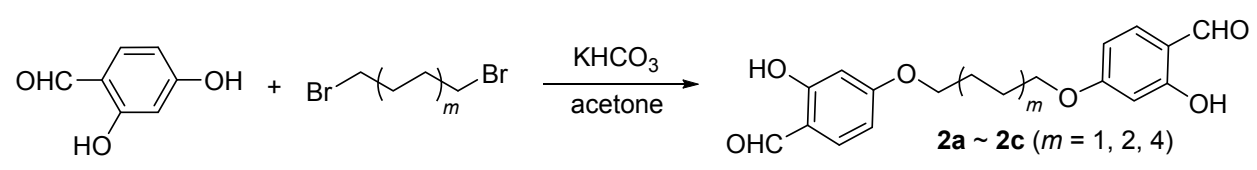

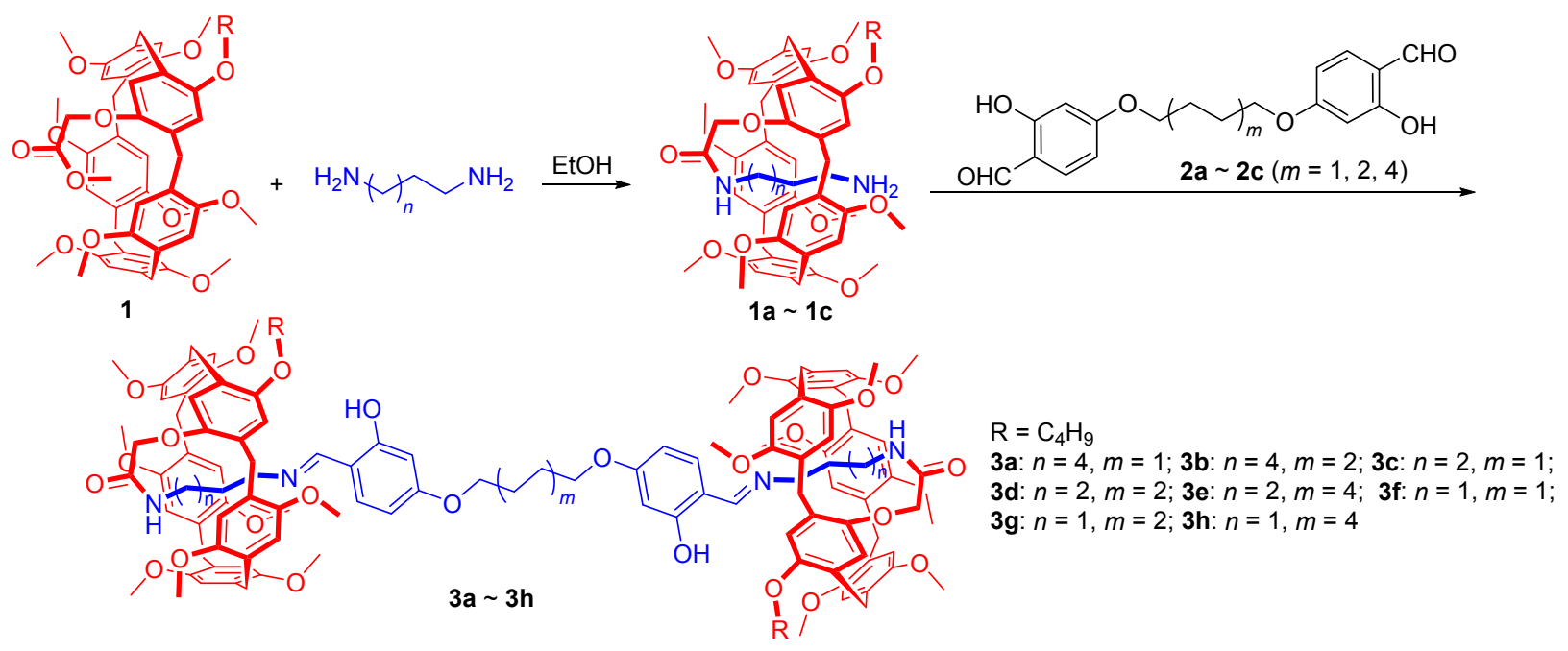

图式 1 双-[1]轮烷 $3 \mathrm{a} \sim 3 \mathrm{~h}$ 合成路线

Scheme 1 Synthesis of bis-[1] rotaxane $\mathbf{3 a} \sim \mathbf{3 h}$ 


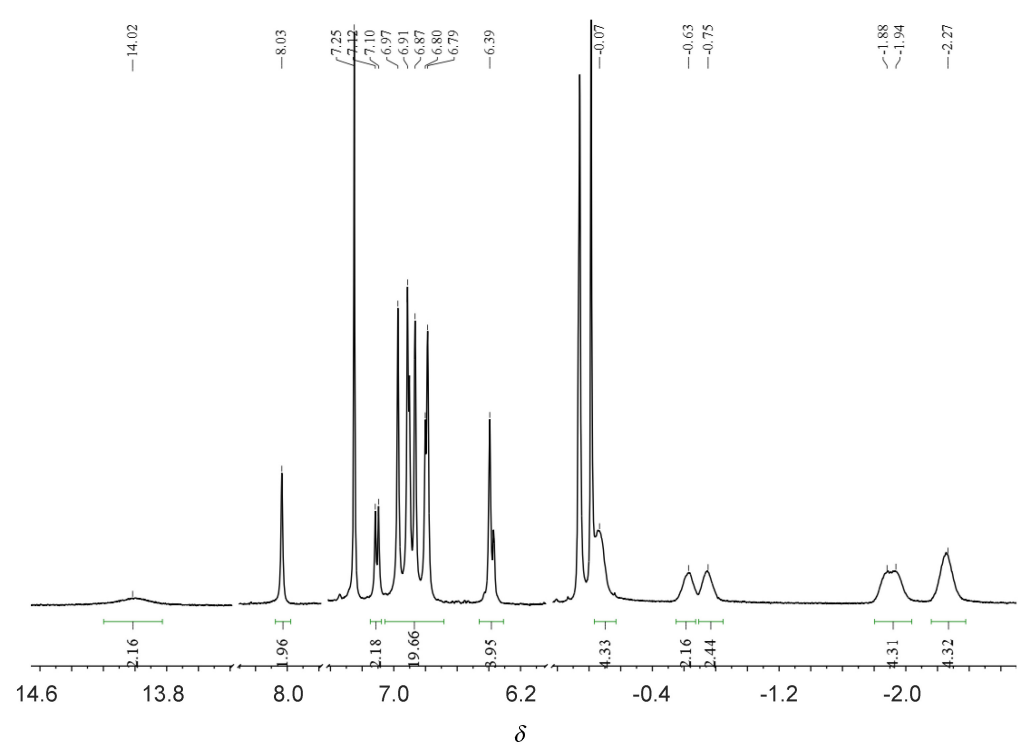

图 1 双[1]轮烷 3a 的核磁氢谱部分图(400 MHz, $\left.298 \mathrm{~K}, \mathrm{CDCl}_{3}\right)$

Figure 1 Partial ${ }^{1} \mathrm{H}$ NMR spectrum $\left(400 \mathrm{MHz}, 298 \mathrm{~K}, \mathrm{CDCl}_{3}\right)$ of bis-[1]rotaxane 3a

表 18 个双[1]轮烷产率及核磁对比

Table 1 Yields of bis[1]rotaxanes and their difference on ${ }^{1} \mathrm{H}$ NMR

\begin{tabular}{ccccc}
\hline Compd. & $n$ & $m$ & Yield/\% & Number of $\mathrm{H}(\delta<0)$ \\
\hline 3a & 4 & 1 & 66.5 & $16\left(\mathrm{CH}_{2}\right)$ \\
3b & 4 & 2 & 54.6 & $16\left(\mathrm{CH}_{2}\right)$ \\
3c & 2 & 1 & 83.4 & $8\left(\mathrm{CH}_{2}\right)$ \\
3d & 2 & 2 & 65.3 & $8\left(\mathrm{CH}_{2}\right)$ \\
3e & 2 & 4 & 64.3 & $8\left(\mathrm{CH}_{2}\right)$ \\
3f & 1 & 1 & 81.1 & $8\left(\mathrm{CH}_{2}\right)$ \\
3g & 1 & 2 & 65.2 & $8\left(\mathrm{CH}_{2}\right)$ \\
3h & 1 & 4 & 52.6 & $8\left(\mathrm{CH}_{2}\right)$ \\
\hline
\end{tabular}

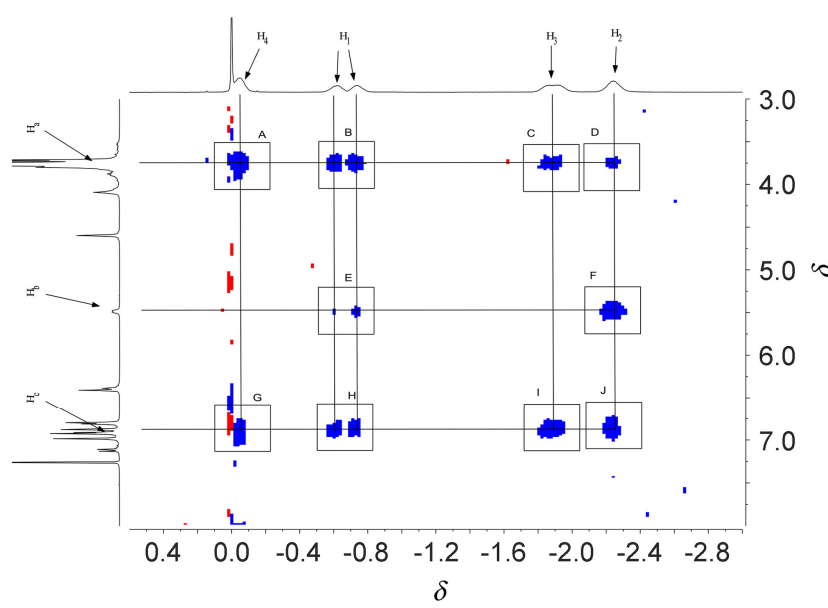

图 2 双[1]轮烷 3a 的 ${ }^{1} \mathrm{H}-{ }^{1} \mathrm{H}$ NOESY 图谱(600 MHz, 298K, $\left.\mathrm{CDCl}_{3}\right)$

Figure $2{ }^{1} \mathrm{H}-{ }^{1} \mathrm{H}$ NOESY spectrum $\left(600 \mathrm{MHz}, 298 \mathrm{~K}, \mathrm{CDCl}_{3}\right)$ of bis-[1] rotaxane 3a

套在烷基二胺的烷基链上.

\section{3 化合物 $3 a$ 的应用}

合成的以席夫碱桥联双柱[5]芳烃为骨架的双-[1]轮
烷具有亚胺和羟基的结构单元，可作为识别位点，通过 氢键相互作用可以作为良好的离子结合位点. 首先研究 了这类化合物对金属离子的选择性 $\left(\mathrm{Cu}^{2+} 、 \mathrm{Co}^{2+} 、 \mathrm{Zn}^{2+}\right.$ 、 $\mathrm{Ni}^{3+} 、 \mathrm{Cd}^{2+}$ 和 $\left.\mathrm{Pb}^{2+}, c=1 \times 10^{-4} \mathrm{~mol} / \mathrm{L}\right)$, 化合物 3a 在 $\mathrm{CH}_{2} \mathrm{Cl}_{2} / \mathrm{MeOH}$ 中的紫外-可见光光谱数据测定(图 3)表 明, 3a 具有两个吸收带，即 $280 \mathrm{~nm}$ 附近的强吸收和 398 $\mathrm{nm}$ 处相对较弱的吸收. 前者是配体芳环上共轭体系的 $\pi-\pi *$ 跃迁产生，后者是由碳氮双键的 $\pi-\pi *$ 跃迁产生. 在 加入金属离子后谱图发生了偏移，席夫碱桥联的双-[1] 轮烷 $3 \mathbf{a}$ 对 $\mathrm{Ni}^{3+} 、 \mathrm{Cd}^{2+}$ 和 $\mathrm{Pb}^{2+}$ 几乎没有识别作用, 而对 $\mathrm{Cu}^{2+}$ 作用最强. 由于 $\mathrm{Cu}^{2+}$ 与席夫碱作用, 席夫碱的紫外 吸收由原来的 $390 \mathrm{~nm}$ 移动至 $350 \mathrm{~nm}$. 通过 3a 与不同浓 度 $\mathrm{Cu}^{2+}$ 的紫外滴定实验，发现两者之间存在着滴定平 衡. 当 $\mathrm{Cu}(\mathrm{OAc})_{2}$ 浓度为配体浓度的 2 倍以后, 谱图不再

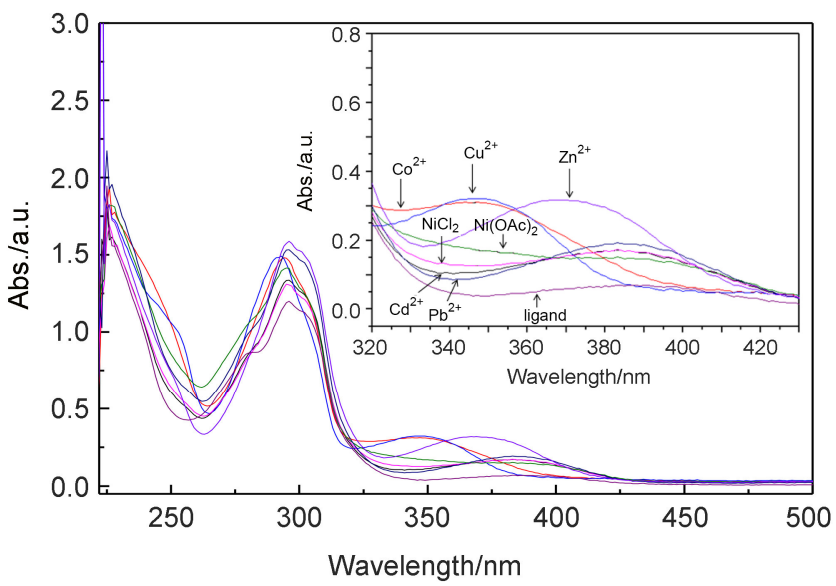

图 3 双 [1]轮烷 3a 与不同金属离子的紫外吸收图

Figure 3 Ultraviolet absorption diagram of bis-[1]rotaxane 3a and different metal ions 
发生变化, 这说明该席夫碱与铜离子之间存在滴定平 衡, 络合比为 $1: 2$ (图 4).

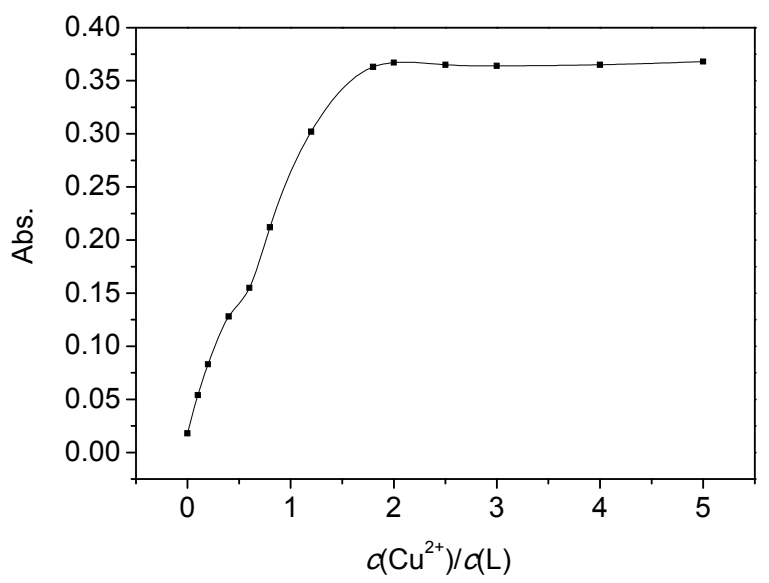

图 4 双 [1]轮烷 $3 \mathrm{a}$ 与不同浓度的 $\mathrm{Cu}^{2+}$ 离子的络合比曲线 Figure 4 Complex ratio curve of bis-[1] rotaxane 3a with differrent concentrations of $\mathrm{Cu}^{2+}$

\section{2 结论}

基于准轮烷的合成, 通过简单的缩合反应, 成功合 成了 8 个以席夫碱桥联的 [5]芳烃为骨架的双-[1]轮烷. 对于较长的桥联链的双柱 [5]芳烃 $\mathbf{3 a} \sim \mathbf{3 h}$, 在溶液中观 察到两个柱 [5]芳烃单元和桥联链存在穿线行为. 此研 究扩展了基于柱[5]芳烃的分子系统, 并为合成自锁柱 [5]芳烃分子拓展了道路. 合成的席夫碱桥联双柱 [5]芳 烃与不同金属离子存在识别作用, 尤其是对 $\mathrm{Cu}^{2+}$ 识别 作用最强, 并以 $1: 2$ 比进行络合, 为检测金属离子传感 器提供材料支持.

\section{3 实验部分}

\section{1 仪器与试剂}

除非另有说明, 所有反应均在开放的气氛中进行, 所有试剂均来自商业来源. 用 Focus X-4 装置测定熔点, 未经校正. 所有产率均以分离提纯后的计算结果. NMR 谱在 Ailent 400 和 AVANCE 600 核磁共振波谱仪上测定, 内标为四甲基硅烷(TMS), 以溶剂信号作为内参. 在 Bruker DPX 600MHz 光谱仪上进行 2D NOESY 实验, 在 Bruker maXis 超高分辨飞行时间质谱仪测定 HR-ESIMS.

\section{2 实验方法}

\section{2 .1 化合物 $\mathbf{2 a} \sim \mathbf{2} \mathrm{c}$ 的合成}

根据报道的方法 ${ }^{[28]}$ 合成双水杨醛化合物 $\mathbf{2 a} \sim \mathbf{2}$.

\section{2 .2 化合物 $\mathbf{3 a} \sim \mathbf{3 h}$ 的合成}

根据报道的方法 ${ }^{[25 \mathrm{a}]}$ 合成酰氨基官能化的柱 [5]芳烃 1a、1b、1c $(0.2 \mathrm{mmol})$, 然后在无水乙醇中加入双水杨
醛类化合物 $\mathbf{2 a} \sim 2 \mathbf{c}(0.1 \mathrm{mmol}), 2 \sim 3 \mathrm{~d}$ 醋酸, 将混合物 加热回流下搅拌 $6 \mathrm{~h}$. 抽滤, 取滤饼, 用乙醇重结晶, 得 到纯产物。

双[1]轮烷柱芳烃 3a: 黄色固体，产率 66.5\%. m.p. $165.5 \sim 167.4{ }^{\circ} \mathrm{C} ;{ }^{1} \mathrm{H}$ NMR $\left(400 \mathrm{MHz}, \mathrm{CDCl}_{3}\right) \delta: 8.06(\mathrm{~s}$, $2 \mathrm{H}, \mathrm{CH}=\mathrm{N}), 7.12(\mathrm{~d}, J=8.3 \mathrm{~Hz}, 2 \mathrm{H}, \mathrm{ArH}), 7.00 \sim 6.78$ $(\mathrm{m}, 20 \mathrm{H}, \mathrm{ArH}), 6.40(\mathrm{~d}, J=9.1 \mathrm{~Hz}, 4 \mathrm{H}, \mathrm{ArH}), 5.48(\mathrm{~d}, J=$ $7.1 \mathrm{~Hz}, 2 \mathrm{H}, \mathrm{NH}), 4.60$ (s, 4H, $\left.\mathrm{CH}_{2}\right), 4.09$ (s, 4H, $\mathrm{CH}_{2}$ ), $3.95 \sim 3.49\left(\mathrm{~m}, 74 \mathrm{H}, 48 \mathrm{OCH}_{3}, 26 \mathrm{CH}_{2}\right), 2.90\left(\mathrm{~s}, 2 \mathrm{H}, \mathrm{CH}_{2}\right)$, 2.59 (t, $\left.J=8.5 \mathrm{~Hz}, 4 \mathrm{H}, \mathrm{CH}_{2}\right), 2.03\left(\mathrm{~s}, 4 \mathrm{H}, \mathrm{CH}_{2}\right), 1.71(\mathrm{dt}$, $\left.J=14.6,7.4 \mathrm{~Hz}, 4 \mathrm{H}, \mathrm{CH}_{2}\right), 1.50$ (q, $J=7.4 \mathrm{~Hz}, 4 \mathrm{H}, \mathrm{CH}_{2}$ ), $0.92\left(\mathrm{t}, J=7.4 \mathrm{~Hz}, 6 \mathrm{H}, \mathrm{CH}_{3}\right),-0.04\left(\mathrm{~s}, 4 \mathrm{H}, \mathrm{CH}_{2}\right),-0.62$ (s, $\left.2 \mathrm{H}, \mathrm{CH}_{2}\right),-0.74\left(\mathrm{~s}, 2 \mathrm{H}, \mathrm{CH}_{2}\right),-1.86\left(\mathrm{~s}, 4 \mathrm{H}, \mathrm{CH}_{2}\right)$, $-2.24\left(\mathrm{~s}, 4 \mathrm{H}, \mathrm{CH}_{2}\right) ;{ }^{13} \mathrm{C}$ NMR $\left(101 \mathrm{MHz}, \mathrm{CDCl}_{3}\right) \delta$ : $168.63,167.59,163.59,162.30,150.89,150.59,150.46$, $150.34,150.31,150.27,150.22,150.08,149.95,146.88$, $132.37,129.30,129.23,128.56,128.41,128.30,128.23$, $127.81,127.15,115.26,114.44,114.03,113.66,112.92$, $112.72,112.63,112.14,112.04,111.74,106.66,102.04$, $68.39,67.48,65.77,56.69,56.18,56.05,55.75,55.71$, $55.63,55.39,55.20,55.12,37.51,32.01,30.68,29.91$, $29.11,29.03,28.70,28.51,27.23,25.89,24.29,22.78$, 19.56, 14.05; IR (KBr) v: 3415, 2936, 1688, 1626, 1500, 1466, 1400, 1296, 1213, 1048, 928, 879, 775, 705, 648 $\mathrm{cm}^{-1}$; HRMS (ESI) calcd for $\mathrm{C}_{128} \mathrm{H}_{155} \mathrm{~N}_{4} \mathrm{O}_{26}\left([\mathrm{M}+\mathrm{H}]^{+}\right)$: 2165.0950, found 2165.0958.

双[1]轮烷柱芳烃 3b: 黄色固体, 产率 54.6\%. m.p. $140.8 \sim 142.3{ }^{\circ} \mathrm{C} ;{ }^{1} \mathrm{H}$ NMR $\left(400 \mathrm{MHz}, \mathrm{CDCl}_{3}\right) \delta: 8.05$ (s, $2 \mathrm{H}, \mathrm{CH}=\mathrm{N}), 7.11(\mathrm{~d}, J=8.3 \mathrm{~Hz}, 2 \mathrm{H}, \mathrm{ArH}), 6.98(\mathrm{~s}, 4 \mathrm{H}$, $\operatorname{ArH}), 6.92$ (d, $J=5.6 \mathrm{~Hz}, 6 \mathrm{H}, \operatorname{ArH}), 6.88$ (s, 4H, ArH), $6.81(\mathrm{~d}, J=3.7 \mathrm{~Hz}, 6 \mathrm{H}, \mathrm{ArH}), 6.39(\mathrm{~d}, J=8.4 \mathrm{~Hz}, 4 \mathrm{H}$, ArH), 5.47 (d, J=7.9 Hz, 2H, NH), 4.59 (s, 4H, $\mathrm{CH}_{2}$ ), 4.03 (t, $\left.J=6.5 \mathrm{~Hz}, 4 \mathrm{H}, \mathrm{CH}_{2}\right), 3.90 \sim 3.67\left(\mathrm{~m}, 74 \mathrm{H}, 48 \mathrm{OCH}_{3}, 26\right.$ $\left.\mathrm{CH}_{2}\right), 2.89$ (s, $\left.2 \mathrm{H}, \mathrm{CH}_{2}\right), 2.59$ (t, $\left.J=8.4 \mathrm{~Hz}, 4 \mathrm{H}, \mathrm{CH}_{2}\right), 1.87$ (t, $\left.J=8.5 \mathrm{~Hz}, 8 \mathrm{H}, \mathrm{CH}_{2}\right), 1.74 \sim 1.67\left(\mathrm{~m}, 4 \mathrm{H}, \mathrm{CH}_{2}\right), 1.49(\mathrm{q}$, $\left.J=7.4 \mathrm{~Hz}, 4 \mathrm{H}, \mathrm{CH}_{2}\right), 0.92\left(\mathrm{t}, J=7.4 \mathrm{~Hz}, 6 \mathrm{H}, \mathrm{CH}_{3}\right),-$ $0.04\left(\mathrm{~s}, 4 \mathrm{H}, \mathrm{CH}_{2}\right),-0.63\left(\mathrm{~s}, 2 \mathrm{H}, \mathrm{CH}_{2}\right),-0.75(\mathrm{~s}, 2 \mathrm{H}$, $\left.\mathrm{CH}_{2}\right),-1.88\left(\mathrm{~d}, J=25.1 \mathrm{~Hz}, 4 \mathrm{H}, \mathrm{CH}_{2}\right),-2.24(\mathrm{~s}, 4 \mathrm{H}$, $\left.\mathrm{CH}_{2}\right) ;{ }^{13} \mathrm{C}$ NMR $\left(101 \mathrm{MHz}, \mathrm{CDCl}_{3}\right) \delta: 168.44,167.58$, $163.68,162.30,150.90,150.59,150.46,150.35,150.32$, $150.28,150.23,150.08,149.96,146.88,132.31,129.30$, $129.28,129.23,128.55,128.53,128.41,128.30,128.23$, $127.81,127.15,115.25,114.43,114.01,113.66,112.92$, $112.73,112.63,112.15,112.06,111.87,111.69,106.73$, $102.01,101.92,68.37,67.88,67.83,65.78,56.82,56.17$, 
$56.04,55.75,55.70,55.63,55.39,55.19,55.12,37.52$, $32.01,30.71,29.92,29.07,29.02,28.70,28.52,27.23$, 26.66, 25.90, 25.86, 24.32, 22.80, 19.56, 14.05; IR (KBr) v: 3410, 2937, 2859, 2036, 1681, 1625, 1504, 1459, 1399, 1296, 1212, 1112, 1043, 928, 873, 845, 775, 711, 647, 546 $\mathrm{cm}^{-1}$; HRMS (ESI) calcd for $\mathrm{C}_{130} \mathrm{H}_{159} \mathrm{~N}_{4} \mathrm{O}_{26}\left([\mathrm{M}+\mathrm{H}]^{+}\right)$: 2193.1271, found 2193.1276.

双[1]轮烷柱芳烃 3c: 黄色固体, 产率 $83.4 \%$. m.p. $194.8 \sim 196.1{ }^{\circ} \mathrm{C} ;{ }^{1} \mathrm{H}$ NMR (400 MHz, $\left.\mathrm{CDCl}_{3}\right) \delta: 13.20$ (s, $2 \mathrm{H}, \mathrm{OH}), 7.23(\mathrm{~s}, 2 \mathrm{H}, \mathrm{ArH}), 7.01 \sim 6.91(\mathrm{~m}, 14 \mathrm{H}, \mathrm{ArH})$, $6.83(\mathrm{~d}, J=9.8 \mathrm{~Hz}, 4 \mathrm{H}, \mathrm{ArH}), 6.77$ (s, 4H, $2 \mathrm{ArH}, 2 \mathrm{CH}=$ $\mathrm{N}), 6.29 \sim 6.22(\mathrm{~m}, 4 \mathrm{H}, \operatorname{ArH}), 5.45(\mathrm{~s}, 2 \mathrm{H}, \mathrm{NH}), 4.59$ (s, $\left.4 \mathrm{H}, \mathrm{CH}_{2}\right), 4.08\left(\mathrm{~s}, 4 \mathrm{H}, \mathrm{CH}_{2}\right), 3.83 \sim 3.73(\mathrm{~m}, 56 \mathrm{H}, 48$ $\left.\mathrm{OCH}_{3}, 8 \mathrm{CH}_{2}\right), 3.71\left(\mathrm{~s}, 6 \mathrm{H}, \mathrm{CH}_{2}\right), 3.65\left(\mathrm{~s}, 4 \mathrm{H}, \mathrm{CH}_{2}\right), 3.58$ (s, 4H, $\left.\mathrm{CH}_{2}\right), 3.40$ (s, 4H, $\left.\mathrm{CH}_{2}\right), 2.87$ (s, 2H, $\left.\mathrm{CH}_{2}\right), 2.04$ (s, $\left.4 \mathrm{H}, \mathrm{CH}_{2}\right), 1.80\left(\mathrm{~s}, 4 \mathrm{H}, \mathrm{CH}_{2}\right), 1.55$ (dd, $J=7.6,3.6 \mathrm{~Hz}, 4 \mathrm{H}$, $\left.\mathrm{CH}_{2}\right), 1.00\left(\mathrm{t}, J=7.5 \mathrm{~Hz}, 6 \mathrm{H}, \mathrm{CH}_{3}\right), 0.29$ (d, $J=43.9 \mathrm{~Hz}$, $\left.4 \mathrm{H}, \mathrm{CH}_{2}\right),-0.92\left(\mathrm{~s}, 4 \mathrm{H}, \mathrm{CH}_{2}\right),-1.91(\mathrm{~d}, J=31.0 \mathrm{~Hz}$, $\left.4 \mathrm{H}, \mathrm{CH}_{2}\right) ;{ }^{13} \mathrm{C} \mathrm{NMR}\left(101 \mathrm{MHz}, \mathrm{CDCl}_{3}\right) \delta: 172.60,167.13$, $164.12,161.56,151.64,150.65,150.61,150.51,150.39$, $150.31,150.26,150.18,149.59,147.61,133.24,130.76$, $129.59,128.63,128.54,128.17,128.15,128.09,127.38$, $127.30,117.94,114.88,113.88,113.78,113.48,113.36$, $112.97,112.83,112.45,111.93,111.37,105.33,102.25$, $70.36,67.26,65.70,55.99,55.96,55.91,55.81,55.75$, $55.61,55.49,55.40,51.15,36.33,32.01,30.48,29.51$, 29.23, 29.04, 28.42, 28.16, 25.97, 25.67, 22.75, 19.48, 14.13; IR (KBr) v: 3415, 2940, 1685, 1624, 1502, 1460, 1399, 1299, 1213, 1112, 1045, 929, 873, 776, 707, 648 $\mathrm{cm}^{-1}$; HRMS (ESI) calcd for $\mathrm{C}_{124} \mathrm{H}_{147} \mathrm{~N}_{4} \mathrm{O}_{26}\left([\mathrm{M}+\mathrm{H}]^{+}\right)$: 2109.0346, found 2109.0337 .

双[1]轮烷柱芳烃 3d: 黄色固体, 产率 64.3\%. m.p. $168.0 \sim 170.2{ }^{\circ} \mathrm{C} ;{ }^{1} \mathrm{H}$ NMR (400 MHz, $\left.\mathrm{CDCl}_{3}\right) \delta: 7.23$ (d, $J=8.5 \mathrm{~Hz}, 2 \mathrm{H}, \mathrm{ArH}), 7.01(\mathrm{~s}, 2 \mathrm{H}, \mathrm{CH}=\mathrm{N}), 7.00 \sim 6.88$ (m, 12H, ArH), 6.83 (d, J=9.2 Hz, 4H, ArH), 6.76 (dd, $J=10.0,2.8 \mathrm{~Hz}, 4 \mathrm{H}, \mathrm{ArH}), 6.25$ (d, $J=10.3 \mathrm{~Hz}, 4 \mathrm{H}, \mathrm{ArH})$, $5.45(\mathrm{~s}, 2 \mathrm{H}, \mathrm{NH}), 4.59\left(\mathrm{~s}, 3 \mathrm{H}, \mathrm{CH}_{2}\right), 4.14\left(\mathrm{~s}, 2 \mathrm{H}, \mathrm{CH}_{2}\right)$, $4.01\left(\mathrm{t}, J=6.5 \mathrm{~Hz}, 4 \mathrm{H}, \mathrm{CH}_{2}\right), 3.96 \sim 3.61(\mathrm{~m}, 64 \mathrm{H}$, $\left.48 \mathrm{OCH}_{3}, 16 \mathrm{CH}_{2}\right), 3.57\left(\mathrm{~s}, 4 \mathrm{H}, \mathrm{CH}_{2}\right), 3.39\left(\mathrm{~s}, 4 \mathrm{H}, \mathrm{CH}_{2}\right)$, $2.86\left(\mathrm{~s}, 2 \mathrm{H}, \mathrm{CH}_{2}\right), 1.88\left(\mathrm{t}, J=6.9 \mathrm{~Hz}, 4 \mathrm{H}, \mathrm{CH}_{2}\right), 1.76(\mathrm{~d}$, $\left.J=7.3 \mathrm{~Hz}, 4 \mathrm{H}, \mathrm{CH}_{2}\right), 1.60\left(\mathrm{~s}, 4 \mathrm{H}, \mathrm{CH}_{2}\right), 1.54$ (dd, $J=7.5$, $\left.3.7 \mathrm{~Hz}, 4 \mathrm{H}, \mathrm{CH}_{2}\right), 1.00\left(\mathrm{t}, J=7.3 \mathrm{~Hz}, 6 \mathrm{H}, \mathrm{CH}_{3}\right), 0.34$ (s, $\left.2 \mathrm{H}, \mathrm{CH}_{2}\right), 0.23\left(\mathrm{~s}, 2 \mathrm{H}, \mathrm{CH}_{2}\right),-0.92$ (d, J=25.7 Hz, 4H, $\left.\mathrm{CH}_{2}\right),-1.90\left(\mathrm{~d}, J=35.9 \mathrm{~Hz}, 4 \mathrm{H}, \mathrm{CH}_{2}\right) ;{ }^{13} \mathrm{C}$ NMR $(101$ $\left.\mathrm{MHz}, \mathrm{CDCl}_{3}\right) \delta: 172.49,167.14,164.23,161.57,151.64$,
$150.65,150.56,150.52,150.39,150.31,150.27,150.19$, $149.60,147.61,133.20,130.75,130.74,129.59,128.63$, $128.53,128.47,128.46,128.19,128.16,128.10,127.38$, $117.90,114.89,113.89,113.70,113.53,113.47,113.39$, $113.00,112.83,112.50,112.48,112.45,111.94,111.31$, $105.43,102.22,70.34,67.69,65.70,55.99,55.96,55.92$, $55.80,55.76,55.57,55.52,55.49,55.39,51.22,51.22$, $36.34,36.33,32.00,29.46,29.18,29.04,28.40,26.00$, $25.65,22.76,19.47,14.11$; IR (KBr) v: 3406, 2940, 2837, 1684, 1625, 1502, 1460, 1399, 1299, 1212, 1044, 928, 877, 776, 707, $649 \mathrm{~cm}^{-1}$; HRMS (ESI) calcd for $\mathrm{C}_{126} \mathrm{H}_{151} \mathrm{~N}_{4} \mathrm{O}_{26}$ $\left([\mathrm{M}+\mathrm{H}]^{+}\right): 2137.0653$, found 2137.0650.

双[1]轮烷柱芳烃 3e: 黄色固体, 产率 65.3\%. m.p. $137.8 \sim 139.5{ }^{\circ} \mathrm{C} ;{ }^{1} \mathrm{H} \mathrm{NMR}\left(400 \mathrm{MHz}, \mathrm{CDCl}_{3}\right) \delta: 13.10$ (s, $2 \mathrm{H}, \mathrm{OH}), 7.22$ (d, J=8.6 Hz, 2H, ArH), 7.00 (s, 2H, $\mathrm{CH}=$ $\mathrm{N}), 6.99 \sim 6.90(\mathrm{~m}, 12 \mathrm{H}, \mathrm{ArH}), 6.82(\mathrm{~d}, J=9.5 \mathrm{~Hz}, 4 \mathrm{H}$, ArH), 6.77 (d, J=3.0 Hz, 4H, ArH), 6.27 $6.20(\mathrm{~m}, 4 \mathrm{H}$, ArH), 5.46 (s, 2H, NH), 4.59 (s, 4H, $\left.\mathrm{CH}_{2}\right), 4.13(\mathrm{~s}, 2 \mathrm{H}$, $\left.\mathrm{CH}_{2}\right), 3.98\left(\mathrm{t}, J=6.6 \mathrm{~Hz}, 4 \mathrm{H}, \mathrm{CH}_{2}\right), 3.87 \sim 3.66(\mathrm{~m}, 60 \mathrm{H}$, $\left.48 \mathrm{OCH}_{3}, 12 \mathrm{CH}_{2}\right), 3.57\left(\mathrm{~s}, 6 \mathrm{H}, \mathrm{CH}_{2}\right), 3.38\left(\mathrm{~s}, 6 \mathrm{H}, \mathrm{CH}_{2}\right)$, $2.86\left(\mathrm{~s}, 2 \mathrm{H}, \mathrm{CH}_{2}\right), 1.81\left(\mathrm{dt}, J=16.0,8.0 \mathrm{~Hz}, 16 \mathrm{H}, \mathrm{CH}_{2}\right)$, $1.51\left(\mathrm{dt}, J=9.2,4.7 \mathrm{~Hz}, 8 \mathrm{H}, \mathrm{CH}_{2}\right), 0.99$ (t, $J=7.4 \mathrm{~Hz}, 6 \mathrm{H}$, $\left.\mathrm{CH}_{3}\right), 0.33\left(\mathrm{~s}, 2 \mathrm{H}, \mathrm{CH}_{2}\right), 0.21\left(\mathrm{~s}, 2 \mathrm{H}, \mathrm{CH}_{2}\right),-0.92(\mathrm{~d}, J=$ $\left.28.4 \mathrm{~Hz}, 4 \mathrm{H}, \mathrm{CH}_{2}\right),-1.92\left(\mathrm{~d}, J=32.5 \mathrm{~Hz}, 4 \mathrm{H}, \mathrm{CH}_{2}\right) ;{ }^{13} \mathrm{C}$ NMR (101 MHz, $\left.\mathrm{CDCl}_{3}\right) \delta: 172.51,167.14,164.30$, $161.53,151.63,150.64,150.51,150.39,150.37,150.29$, $150.25,150.18,149.57,147.58,133.16,130.73,129.57$, $128.62,128.53,128.51,128.45,128.15,128.08,127.36$, $127.29,117.89,114.86,113.86,113.48,113.35,112.95$, $112.82,112.44,111.92,111.24,105.46,102.20,70.32$, $67.85,65.68,55.98,55.95,55.90,55.80,55.74,55.48$, $55.39,51.16,36.34,32.00,30.47,29.58,29.46,29.20$, $28.39,28.13,26.12,25.65,22.75,19.46,14.11$; IR (KBr) $v: 3406,2936,2857,2480,2035,1682,1625,1503,1458$, 1398, 1298, 1212, 1112, 1043, 929, 875, 776, 711, 648, $550 \mathrm{~cm}^{-1}$; HRMS (ESI) calcd for $\mathrm{C}_{130} \mathrm{H}_{159} \mathrm{~N}_{4} \mathrm{O}_{26}([\mathrm{M}+$ $\mathrm{H}^{+}$): 2193.1293, found 2193.1276.

双[1]轮烷柱芳烃 3f: 黄色固体, 产率 $81.1 \%$. m.p. 206.2 208.6 ${ }^{\circ} \mathrm{C} ;{ }^{1} \mathrm{H}$ NMR (400 MHz, $\left.\mathrm{CDCl}_{3}\right) \delta: 13.01$ (s, $2 \mathrm{H}, \mathrm{OH}), 7.18$ (d, $J=8.7 \mathrm{~Hz}, 2 \mathrm{H}, \mathrm{ArH}), 7.06 \sim 6.79(\mathrm{~m}$, $18 \mathrm{H}, \operatorname{ArH}), 6.69(\mathrm{~s}, 2 \mathrm{H}, \operatorname{ArH}), 6.32(\mathrm{~s}, 2 \mathrm{H}, \mathrm{CH}=\mathrm{N})$, $6.30 \sim 6.22(\mathrm{~m}, 4 \mathrm{H}, \mathrm{ArH}), 4.51\left(\mathrm{~s}, 4 \mathrm{H}, \mathrm{CH}_{2}\right), 4.32(\mathrm{~d}, J=$ $8.7 \mathrm{~Hz}, 2 \mathrm{H}, \mathrm{NH}), 4.17 \sim 3.22\left(\mathrm{~m}, 80 \mathrm{H}, 48 \mathrm{OCH}_{3}, 32 \mathrm{CH}_{2}\right)$, $2.03\left(\mathrm{~s}, 4 \mathrm{H}, \mathrm{CH}_{2}\right), 1.82\left(\mathrm{~d}, J=8.4 \mathrm{~Hz}, 4 \mathrm{H}, \mathrm{CH}_{2}\right), 1.58 \sim$ $1.53\left(\mathrm{~m}, 4 \mathrm{H}, \mathrm{CH}_{2}\right), 1.00\left(\mathrm{t}, J=7.4 \mathrm{~Hz}, 6 \mathrm{H}, \mathrm{CH}_{3}\right)$, - 
$0.05 \sim-0.20\left(\mathrm{~m}, 4 \mathrm{H}, \mathrm{CH}_{2}\right),-1.71\left(\mathrm{~s}, 2 \mathrm{H}, \mathrm{CH}_{2}\right),-1.88$ $\left(\mathrm{s}, 2 \mathrm{H}, \mathrm{CH}_{2}\right) ;{ }^{13} \mathrm{C}$ NMR (101 MHz, $\left.\mathrm{CDCl}_{3}\right) \delta: 170.35$, $166.77,163.59,162.31,151.46,150.84,150.56,150.49$, $150.21,150.13,149.71,148.56,132.93,131.73,129.86$, $129.22,128.55,128.44,128.17,127.87,127.78,125.85$, $118.09,114.13,113.95,113.93,113.60,113.37,113.21$, $112.39,112.24,111.57,105.50,101.90,70.37,67.29$, $65.95,56.10,55.95,55.72,55.61,55.45,55.20,55.18$, 55.02 , 50.86, 36.06, 31.92, 30.08, 28.62, 28.20, 27.02, 25.97, 19.47, 14.05; IR (KBr) v: 3407, 2934, 1685, 1627, 1499, 1465, 1399, 1296, 1213, 1045, 928, 880, 775, 705, $648 \mathrm{~cm}^{-1}$; HRMS (ESI) calcd for $\mathrm{C}_{122} \mathrm{H}_{143} \mathrm{~N}_{4} \mathrm{O}_{26}([\mathrm{M}+$ $\mathrm{H}^{+}$): 2081.0058, found 2081.0024.

双 [1] 轮烷柱芳烃 3g: 黄色固体, 产率 $65.2 \%$. m.p.154.7 $156.2{ }^{\circ} \mathrm{C}$; ${ }^{1} \mathrm{H}$ NMR $\left(400 \mathrm{MHz}, \mathrm{CDCl}_{3}\right) \delta$ : 13.00 (s, 2H, OH), 7.16 (d, J=8.6 Hz, 2H, ArH), 7.04 (d, $J=4.8 \mathrm{~Hz}, 4 \mathrm{H}, \mathrm{ArH}), 7.01 \sim 6.91(\mathrm{~m}, 8 \mathrm{H}, \mathrm{ArH}), 6.87$ (s, $2 \mathrm{H}, \mathrm{ArH}), 6.81$ (d, J=6.8 Hz, 4H, ArH), 6.68 (s, 2H, ArH), $6.32(\mathrm{~s}, 2 \mathrm{H}, \mathrm{CH}=\mathrm{N}), 6.30 \sim 6.19(\mathrm{~m}, 4 \mathrm{H}, \mathrm{ArH}), 4.50(\mathrm{~s}$, $\left.4 \mathrm{H}, \mathrm{CH}_{2}\right), 4.32(\mathrm{~d}, J=8.5 \mathrm{~Hz}, 2 \mathrm{H}, \mathrm{NH}), 4.12\left(\mathrm{~s}, 2 \mathrm{H}, \mathrm{CH}_{2}\right)$, $4.07 \sim 3.49\left(\mathrm{~m}, 66 \mathrm{H}, 48 \mathrm{OCH}_{3}, 18 \mathrm{CH}_{2}\right), 3.42\left(\mathrm{~s}, 6 \mathrm{H}, \mathrm{CH}_{2}\right)$, $3.26\left(\mathrm{~s}, 6 \mathrm{H}, \mathrm{CH}_{2}\right), 1.84$ (d, $\left.J=16.8 \mathrm{~Hz}, 8 \mathrm{H}, \mathrm{CH}_{2}\right), 1.55$ (q, $\left.J=7.5 \mathrm{~Hz}, 8 \mathrm{H}, \mathrm{CH}_{2}\right), 1.00\left(\mathrm{t}, J=7.4 \mathrm{~Hz}, 6 \mathrm{H}, \mathrm{CH}_{3}\right)$, $0.07 \sim-0.21\left(\mathrm{~m}, 4 \mathrm{H}, \mathrm{CH}_{2}\right),-1.72\left(\mathrm{~s}, 2 \mathrm{H}, \mathrm{CH}_{2}\right),-1.89$ $\left(\mathrm{s}, 2 \mathrm{H}, \mathrm{CH}_{2}\right) ;{ }^{13} \mathrm{C}$ NMR $\left(101 \mathrm{MHz}, \mathrm{CDCl}_{3}\right) \delta: 221.34$, $170.70,166.82,163.83,162.29,151.47,150.85,150.58$, $150.51,150.21,150.15,149.71,148.55,132.96,131.71$, $129.87,129.22$, 128.59, 128.46, 128.20, 127.86, 127.81, $126.50,125.85,118.10,114.19,113.94,113.62,113.40$, $113.23,112.39,112.23,112.18,111.41,106.85,105.61$, $101.90,70.39,67.73,65.93,56.16,55.96,55.73,55.62$, $55.48,55.21,55.07,50.68,36.06,32.04,31.92,30.09$, 29.19, 28.60, 28.18, 27.01, 25.99, 19.47, 14.05; IR (KBr) $v: 3391,2935,1681,1627,1499,1465,1399,1297,1213$, 1045, 927, 881, 855, 774, 702, $648 \mathrm{~cm}^{-1}$; HRMS (ESI) calcd for $\mathrm{C}_{124} \mathrm{H}_{147} \mathrm{~N}_{4} \mathrm{O}_{26}\left([\mathrm{M}+\mathrm{H}]^{+}\right): 2109.0387$, found 2109.0337.

双[1]轮烷柱芳烃 3h: 黄色固体, 产率 52.6\%. m.p. $142.8 \sim 144.9{ }^{\circ} \mathrm{C} ;{ }^{1} \mathrm{H}$ NMR (400 MHz, $\left.\mathrm{CDCl}_{3}\right) \delta: 12.99$ (s, $2 \mathrm{H}, \mathrm{OH}), 7.14(\mathrm{~d}, J=8.7 \mathrm{~Hz}, 2 \mathrm{H}, \mathrm{ArH}), 7.05 \sim 6.90(\mathrm{~m}$, 12H, ArH), 6.85 (s, 2H, ArH), 6.79 (d, J=7.7 Hz, 4H, $\operatorname{ArH}), 6.67(\mathrm{~s}, 2 \mathrm{H}, \mathrm{ArH}), 6.30(\mathrm{~s}, 2 \mathrm{H}, \mathrm{CH}=\mathrm{N}), 6.25(\mathrm{dd}$, $J=8.6,2.4 \mathrm{~Hz}, 2 \mathrm{H}, \mathrm{ArH}), 6.20$ (d, $J=2.2 \mathrm{~Hz}, 2 \mathrm{H}, \mathrm{ArH})$, $4.49\left(\mathrm{~s}, 4 \mathrm{H}, \mathrm{CH}_{2}\right), 4.30(\mathrm{~d}, J=7.7 \mathrm{~Hz}, 2 \mathrm{H}, \mathrm{NH}), 4.10$ (s, $\left.2 \mathrm{H}, \mathrm{CH}_{2}\right), 4.02 \sim 3.48\left(\mathrm{~m}, 66 \mathrm{H}, 48 \mathrm{OCH}_{3}, 18 \mathrm{CH}_{2}\right), 3.41(\mathrm{~s}$,
6H, $\left.\mathrm{CH}_{2}\right), 3.24\left(\mathrm{~s}, 6 \mathrm{H}, \mathrm{CH}_{2}\right), 1.81\left(\mathrm{t}, J=7.6 \mathrm{~Hz}, 8 \mathrm{H}, \mathrm{CH}_{2}\right)$, $1.51\left(\mathrm{dq}, J=14.0,7.3 \mathrm{~Hz}, 8 \mathrm{H}, \mathrm{CH}_{2}\right), 1.37\left(\mathrm{~s}, 8 \mathrm{H}, \mathrm{CH}_{2}\right)$, $0.98\left(\mathrm{t}, J=7.4 \mathrm{~Hz}, 6 \mathrm{H}, \mathrm{CH}_{3}\right),-0.15(\mathrm{dd}, J=20.0,11.4$ $\left.\mathrm{Hz}, 4 \mathrm{H}, \mathrm{CH}_{2}\right),-1.73\left(\mathrm{~s}, 2 \mathrm{H}, \mathrm{CH}_{2}\right),-1.90\left(\mathrm{~s}, 2 \mathrm{H}, \mathrm{CH}_{2}\right)$; ${ }^{13} \mathrm{C}$ NMR $\left(101 \mathrm{MHz}, \mathrm{CDCl}_{3}\right) \delta: 170.33,166.79,164.30$, $163.78,163.20,162.30,151.45,150.84,150.54,150.50$, $150.19,150.13,149.69,148.53,132.86,132.51,131.68$, $129.84,129.21,128.54,128.44,128.16,127.84,127.77$, $126.49,125.83,118.02,114.12,113.92,113.59,113.36$, $113.21,112.38,112.23,112.12,112.09,111.44,106.86$, 105.64, 101.86, 101.71, 77.37, 70.34, 68.07, 67.90, 65.93, $56.11,55.95,55.72,55.61,55.44,55.19,55.03,50.88$, $36.09,32.05,31.92,30.09,29.58,29.50,29.47,29.41$, $29.36,29.22,29.20,29.08,28.63,28.61,28.17,27.01$, 26.12, 26.08, 26.00, 19.47, 14.06; IR (KBr) v: 3397, 2933, $1683,1626,1500,1465,1399,1297,1213,1045,928,880$, $775,703,648 \mathrm{~cm}^{-1}$; HRMS (ESI) calcd for $\mathrm{C}_{128} \mathrm{H}_{154} \mathrm{~N}_{4} \mathrm{O}_{26}$ $\left([\mathrm{M}+\mathrm{H}]^{+}\right): 2165.0966$, found 2165.0958.

辅助材料(Supporting Information) 化合物 $\mathbf{3 a} \sim \mathbf{3 h}$ 的 HRMS、 ${ }^{1} \mathrm{H}$ NMR 和 ${ }^{13} \mathrm{C}$ NMR 图谱. 这些材料可以免费 从本刊网站(http://sioc-journal.cn/)上下载.

\section{References}

[1] Krakowiak, K. E.; Bradshaw, J.; Zamecka-Krakowiak, D. J. Chem. Rev. 1989, 89, 929

[2] (a) Lehn, J. M. Science 1993, 260, 1762.

(b) Chen, Y.; Sun, S.; Lu, D.; Shi, Y.; Yao, Y. Chin. Chem. Lett. 2019, 30, 27 .

(c) He, M.; Chen, L.; Jiang, B.; Tan, H.; Yang, H. Chin. Chem. Lett., 2019, 30, 131.

[3] Leininger, S.; Olenyuk, B.; Stang, P. J. Chem. Rev. 2000, 100, 853.

[4] (a) Stoddart, J. F. Angew. Chem. Int. Ed. 2017, 56, 11094. (b) Lohmann, F.; Weigandt, J.; Valero, J.; Famulok, M. Angew. Chem. Int. Ed. 2014, 53, 10372.

(c) Fahrenbach, A. C. Acc. Chem. Res. 2014, 47, 482.

(d) Fahrenbach, A. C.; Bruns, C. J.; Cao, D.; Stoddart, J. F. Acc. Chem. Res. 2012, 45, 1581

(e) Weng, G.-H.; Zhu, B.; Ye, Y.; Li, S. Chin. J. Org. Chem. 2015, 35, 309 (in Chinese).

(翁官欢, 朱涁, 叶杨, 李世军, 有机化学, 2015, 35, 309.)

(f) Wang, H.; Kan, J.; Bian, B.; Chen, Q.; Tao, Z.; Xiao, X. Chin. J. Org. Chem. 2018, 38, 3094 (in Chinese).

(王海燕，阚京兰，边炳，陈青，陶朱，肖昕，有机化学，2018，38， 3094.)

(g) Huo, B.; Li, B.; Su, H.; Zeng, X.; Xu, K.; Cui, L. Chin. J. Org. Chem. 2019, 39, 1990 (in Chinese).

(霍博超，李斌，苏杭，曾宪强，徐凯迪，崔雷，有机化学，2019, 39, 1990.)

[5] (a) Amabilino, D. B.; Perez-Garcia, L. Chem. Soc. Rev. 2009, 38, 1562.

(b) Ma, X.; Tian, H. Chem. Soc. Rev. 2010, 39, 70.

(c) Forgan, R. S.; Sauvage, J. P.; Stoddart, J. F. Chem. Rev. 2011, 111,5434 .

(d) Dongen, S. F. M. van; Cantekin, S.; Elemans, J. A. A. W.; Rowan, A. E.; Nolte, R. J. M. Chem. Soc. Rev. 2014, 43, 99. 
(e) Xue, M.; Yang, Y.; Chi, X.; Yan, X.; Huang, F. Chem. Rev. 2015, 115,7398

[6] (a) Cao, J.; Ma, X.; Min, M. Chem. Commun. 2014, 50, 3224

(b) Leigh, D. A.; Marcos, V.; Nalbantoglu, T. J. Am. Chem. Soc. 2017, 139, 7104.

(c) Niu, Z. B.; Gibson, H. W. Chem. Rev. 2009, 109, 6024.

(d) Raymo, F. M.; Stoddart, J. F. Chem. Rev. 1999, 99, 1643.

(e) Balzani, V.; Gómez-López, M.; Stoddart, J. F. Acc. Chem. Res. 1998, 31, 405

(f) Harada, A.; Takashima, Y.; Yamaguchi, H. Chem. Soc. Rev. 2009, 38, 875 .

[7] Ashton, P. R.; Goodnow, T. T.; Kaifer, A. E. Angew. Chem. Int. Ed. 1989, 28,1396

[8] (a) Silvi, S.; Venturi, M.; Credi, A. J. Mater. Chem. 2009, 19, 2279 (b) Crowley, J. D.; Goldup, S. M.; Lee, A. L.; Leigh, D. A.; McBurney, R. T. Chem. Soc. Rev. 2009, 38, 1530.

(c) Anelli, P. L.; Spencer, N.; Stoddart, J. F. J. Am. Chem. Soc. 1991, 113, 5131 .

[9] Tan, L. L.; Li, H.; Qiu, Y. C. Chem. Sci. 2015, 6, 1640.

[10] (a) Mandal, A. K.; Gangopadhyay, M.; Das, A. Chem. Soc. Rev. 2015, 44, 663.

(b) Qu, D. H.; Wang, Q. C.; Zhang, Q. W.; Ma, X.; Tian, H. Chem. Rev. 2015, 115, 7543 .

[11] Wang, Y.; Tian, Y.; Chen, Y. Z. Chem. Commun. 2018, 54, 7991.

[12] Ma, L.; Wang, S.; Li, C. Chem. Commun. 2018, 54, 2405.

[13] Wu, D.; Li, Y.; Shen, J. Chem. Commun. 2018, 54, 8198.

[14] (a) Liu, Y.; Chipot, C.; Shao, X.; Cai, W. J. Phys. Chem. C 2014, 118,19380 .

(b) Li, H.; Li, X.; Ågren, H.; Qu, D. H. Org. Lett. 2014, 16, 4940.

(c) Cao, J.; Ma, X.; Min, M.; Cao, T.; Wu, S.; Tian, H. Chem. Commun. 2014, 50, 3224.

(d) Li, H.; Zhang, J. N.; Zhou, W.; Zhang, H.; Zhang, Q.; Qu, D. H.; Tian, H. Org. Lett. 2013, 15, 3070.

(e) Yamauchi, K.; Miyawaki, A.; Takashima, Y.; Yamaguchi, H.; Harada, A. Org. Lett. 2010, 12, 1284.

(f) Miyawaki, A.; Kuad, P.; Takashima, Y.; Yamaguchi, H.; Harada, A. J. Am. Chem. Soc. 2008, 130, 17062.

(g) Liu, Y.; Yang, Z. X.; Chen, Y. J. Org. Chem. 2008, 73, 5298.

(h) Franchi, P.; Fanì, M.; Mezzina, E.; Lucarini, M. Org. Lett. 2008,

$10,190$.

(i) Hiratani, K.; Kaneyama, M.; Nagawa, Y.; Koyama, E.; Kanesato, M. J. Am. Chem. Soc. 2004, 126, 13568.

[15] (a) Ogoshi, T.; Kanai, S.; Fujinami, S.; Yamagishi, T. A.; Nakamoto, Y. J. Am. Chem. Soc. 2008, 130, 50223.

(b) Cao, D.; Kou, Y.; Liang, J.; Chen, Z.; Wang, L.; Meier, H. Angew. Chem. Int. Ed. 2009, 48, 9721.

(c) Xue, M.; Yang, Y.; Chi, X.; Zhang, Z.; Huang, F. Acc. Chem. Res. 2012, 45, 1294

(d) Cragg, P. J.; Sharma, K. Chem. Soc. Rev. 2012, 41, 597.

(e) Ogoshi, T.; Yamagishi, T. Chem. Commun. 2014, 50, 4776.

(f) Strutt, N. L.; Zhang, H.; Schneebeli, S. T.; Stoddart, J. F. Acc. Chem. Res. 2014, 47, 2631.

(g) Yue, S.; Zhou, Y.; Yao, Y.; Xue, M. Acta Chim. Scinica 2014, 72, 1053 (in Chinese)

(岳诗雨, 周玉娟, 姚勇, 薛敏, 化学学报, 2014, 72, 1053.)

[16] (a) Li, C.; Chen, S.; Li, J.; Han, K.; Xu, M.; Hu, B.; Yu, Y.; Jia, X. Chem. Commun. 2011, 47, 11294.

(b) Hu, X. B.; Chen, Z.; Chen, L.; Zhang, L.; Hou, J. L.; Li, Z. T. Chem. Commun. 2012, 48, 10999.

(c) Hu, X. Y.; Wu, X.; Duan, Q.; Xiao, T.; Lin, C.; Wang, L. Org. Lett. 2012, 14, 4826

(d) Xu, J. F.; Chen, Y. Z.; Wu, L. Z.; Tung, C. H.; Yang, Q. Z. Org. Lett. 2013, 15, 6148. (e) Li, H.; Chen, D. X.; Sun, Y. L.; Zheng, Y. B.; Tan, L. L.; Weiss, P. S.; Yang, Y. W. J. Am. Chem. Soc. 2013, 135, 1570.

(f) Yang, Y. W.; Cao, D. Chin. J. Chem. 2015, 33, 303

(g) Han, C.; Zhang, Z.; Chi, X.; Zhang, M.; Yu, G.; Huang, F. Acta Chim. Sinica 2012, 70, 1775 (in Chinese).

(韩成友, 张子涁, 池小东, 张明明, 喻国灿, 黄飞鹤, 化学学报, 2012, 70, 1775.)

[17] (a) Zhang, Z.; Luo, Y.; Chen, J.; Dong, S.; Yu, Y.; Ma, Z.; Huang, F. Angew. Chem. Int. Ed. 2011, 123, 1433

(b) Hu, X. B.; Chen, Z.; Tang, G.; Hou, J. L.; Li, Z. T. J. Am. Chem. Soc. 2012, 134, 8384.

(c) Chen, L.; Si, W.; Zhang, L.; Tang, G.; Li, Z. T.; Hou, J. L. J. Am. Chem. Soc. 2013, 135, 2152.

(d) Cao, Y.; Hu, X. Y.; Li, Y.; Zou, X.; Xiong, S.; Lin, C.; Shen, Y. Z.; Wang, L. J. Am. Chem. Soc. 2014, 136, 10762

(e) Chen, H.; Fan, J.; Hu, X.; Ma, J.; Wang, S.; Li, J.; Yu, Y.; Jia, X.; Li, C. Chem. Sci. 2015, 6, 197.

(f) Chen, R.; Jiang, H.; Gu, H.; Zhou, Q.; Wu, J.; Chen, D.; Zhang, J. Chem. Commun. 2015, 51, 12220.

(g) Wu, X.; Duan, Q.; Ni, M.; Hu, X.; Wang, L. Chin. J. Org. Chem. 2014, 34, 437 (in Chinese).

(吴旋，段群鹏，倪梦飞，胡晓玉，王乐勇，有机化学，2014，34, 437.)

[18] (a) Zhang, Z.; Xia, B.; Han, C.; Yu, Y.; Huang, F. Org. Lett. 2010, $12,3285$.

(b) Li, C.; Zhao, L.; Li, J.; Ding, X.; Chen, S.; Zhang, Q.; Yu, Y.; Jia, X. Chem. Commun. 2010, 46, 9016.

(c) Zhang, H.; Liu, Z.; Liu, F.; Hao, A. Chin. J. Org. Chem. 2012, 32,219 .

(d) Zhang, H.; Zhao, Y. Chem. Eur. J. 2013, 19, 16862.

(e) Wang, Y.; Xu, J. F.; Chen, Y. Z.; Niu, L. Y.; Wu, L. Z.; Tung, C. H.; Yang, Q. Z. Chem. Commun. 2014, 50, 7001.

(f) Wang, Y.; Ping, G.; Li, C. Chem. Commun. 2016, 52, 9858.

(g) Jiang, S.; Han Y.; Sun, J.; Yan, C. G. Tetrahedron 2017, 73, 5107.

(h) Sun, Y.; Fu, W.; Chen, C.; Wang, J.; Yao, Y. Chem. Commun. 2017, 53, 3725.

[19] Ogoshi, T.; Demachi, K.; Kitajima, K.; Yamagishi, T. A. Chem. Commun. 2011, 47, 7164.

[20] Chen, Y.; Cao, D.; Wang, L.; He, M.; Zhou, L.; Schollmeyer, D.; Meier, H. Chem. Eur. J. 2013, 19, 7064.

[21] Xia, B. Y.; Xue, M. Chem. Commun. 2014, 50, 1021.

[22] Cheng, M.; Wang, Q.; Cao, Y. Tetrahedron Lett. 2016, 57, 4133.

[23] Sun, C. L.; Xu, J. F.; Chen, Y. Z. Chin. Chem. Lett. 2015, 26, 843.

[24] Li, S. H.; Zhang, H. Y.; Xu, X.; Liu, Y. Nat. Commun. 2015, 6, 7590.

[25] (a) Han, Y.; Huo, G. F.; Sun, J.; Xie, Y. J.; Yan, C. G.; Zhao, Y.; Wu, X.; Lin, C.; Wang, L.; Sci. Rep. 2016, 6, 28748.

(b) Han, Y.; Huo, G. F.; Sun, J.; Yan, C. G.; Lu, Y.; Lin, C.; Wang, L. Supramol. Chem. 2017, 29, 547.

[26] Cheng, M.; Wang, Q.; Cao, Y.; Pan, Y.; Yang, Z.; Jiang, J.; Wang, L. Tetrahedron Lett. 2016, 57, 4133.

[27] Zhao, L-L.; Han, Y.; Yan, C.-G. Chin. Chem. Lett. 2019, doi: 10.1016/j.cclet.2019.04.024.

[28] Cerrada. L.; Pinol, M.; Serrano, L. J. J. Polym. Sci., Part A: Polym. Chem. 1996, 34, 2603.

[29] (a) Huo, G. F.; Han, Y.; Sun, J. J. Incl. Phenom. Macrocycl. Chem. 2016, 86, 231.

(b) Jiang, S.; Han, Y.; Sun, J. Tetrahedron 2017, 73, 5107.

(c) Yin, C. B.; Han, Y.; Huo, G. F. Chin. Chem. Lett. 2017, $28,431$.

(d) Jiang, S.; Han, Y.; Cheng, M. New J. Chem. 2018, 42, 7603.

(e) Han, Y.; Xu, L. M.; Nie, C. Y. Beilstein J. Org. Chem. 2018, 14, 1660.

(Li, L.; Fan, Y.) 